\title{
Parathyroid Carcinoma: A Case Report and Review of the Literature
}

\author{
R Forde $^{1}$, A Mitchell ${ }^{1}$, K Duncan ${ }^{1}$, TN Gibson ${ }^{2}$
}

\section{INTRODUCTION}

Primary hyperparathyroidism is the most frequent cause of asymptomatic hypercalcaemia in ambulatory patients (1). Parathyroid carcinoma (PCA) accounts for $5 \%$ of all the cases of primary and it has a variable prognosis (2). There is no published literature in the West Indies regarding the management of parathyroid carcinoma and this is the fourth case seen in our department of surgery for over 40 years. This report highlights an interesting presentation of parathyroid carcinoma and aims to review the current diagnosis and treatment of this disease entity.

\section{CASE REPORT}

A 67-year old woman with a history of diabetes melitus and hypertension and no family history of cancer was found on routine investigation to have hypercalcaemia, $3.51 \mathrm{mmol} / \mathrm{L}$ (normal range: $2.11-2.62 \mathrm{mmol} / \mathrm{L}$ ), as an outpatient by her general practitioner. She was asymptomatic. Work-up for the cause of her hypercalcaemia included a parathyroid hormone assay and phosphorus level which were found to be 26.3 $\mathrm{pmol} / \mathrm{L}$ (normal range: $1.3-7.6 \mathrm{pmol} / \mathrm{L}$ ) and $0.5 \mathrm{mmol} / \mathrm{L}$ (normal range: $0.7-1.5 \mathrm{mmol} / \mathrm{L})$, respectively. Thyroid ultrasound revealed a $3.2 \times 2.2 \mathrm{~cm}$ hypoechoic solid nodule adjacent to the posterior margin of the left lobe of the thyroid gland. There were no calcifications and the nodule was hypovascular. The conclusion was parathyroid adenoma versus carcinoma. There was no cervical lymphadenopathy.

An abdominal ultrasound was also requested by the attending physician which revealed a non-obstructing right lower pole renal calculus. An ultrasound-guided fine needle aspiration cytology (FNAC) of this nodule revealed thyroid epithelial cells with enlarged nuclei which formed acini, sheets and papillary buds. There were no nuclear grooves and holes identified and there was a rich branching fibrovascular core. The conclusion was suspicious for follicular variant of papillary carcinoma. A parathyroid technetium-99 sestamibi scan showed persistent marked uptake in the upper half of the left lobe after two and three hours which was diagnostic for a parathyroid neoplasm. Her creatinine clearance, 24-hour urine calcium and thyroid function test were normal. She was re-

Keywords: Hypercalcaemia, hyperparathyroidism, parathyroid cancer

From: ${ }^{1}$ Ear Nose Throat Division, Department of Surgery, Radiology, Anaesthesia and Intensive Care and ${ }^{2}$ Department of Pathology, The University of the West Indies, Kingston 7, Jamaica.

Correspondence: Dr R Forde, Department of Surgery, Radiology, Anaesthesia and Intensive Care, The University of the West Indies, Kingston 7 , Jamaica, West Indies. E-mail: royantonioforde@hotmail.com; draforde@ gmail.com ferred to the Ear Nose and Throat (ENT) Clinic by her general practitioner. On examination, the only significant finding was a $3 \mathrm{~cm}$ left-sided anterior neck mass in the region of the thyroid which moved on swallowing. She was consented for bilateral neck exploration and total thyroidectomy. Prior to surgery, she was started on saline diuresis and oral furosemide by the endocrinology team. Her preoperative calcium was 2.89 $\mathrm{mmol} / \mathrm{L}$.

At surgery, she was found to have a firm, irregular $5 \times 5$ $\mathrm{cm}$ mass involving the left upper lobe of the thyroid which was encasing the cricopharyngeus and recurrent laryngeal nerve and invaded the muscular layer of the upper oesophagus (Fig. 1).

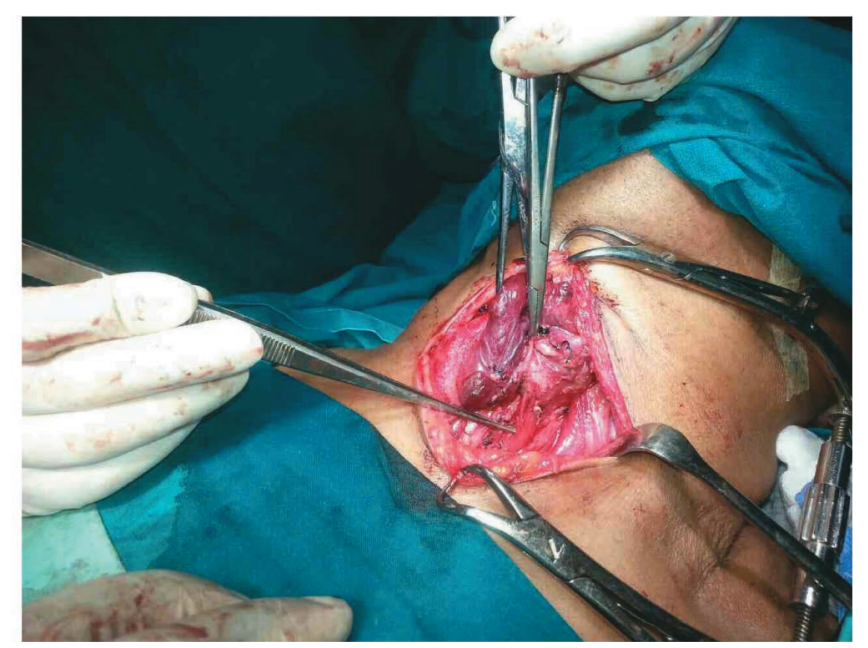

Fig.1: Left lateral view of the neck of a patient undergoing parathyroidectomy. The left thyroid lobe is rotated and the parathyroid mass is seen intimately involving the upper oesophagus (dissecting forceps) and recurrent laryngeal nerve (artery forceps).

Frozen section analysis of the left inferior parathyroid revealed fibro-adipose tissue only, but subsequent deeper levels showed a minute portion of normocellular parathyroid gland. The mass was dissected free of the left recurrent laryngeal nerve and the pharyngoesophagus and the surgery was completed with a total thryoidectomy preserving two normal parathyroids on the right. The mass and the total thyroidectomy specimen were sent for histopathological evaluation. She had an uneventful postoperative period and her calcium stabilized at $2.23 \mathrm{mmol} / \mathrm{L}$. The total thyroidectomy specimen weighed $28.5 \mathrm{~g}$ and serial sectioning revealed a $2.8 \times 2.0 \times 1.3$ $\mathrm{cm}$ circumscribed, yellow-white mass within the upper pole of the left lobe. The mass abutted the thyroid gland capsule. The remainder of the thyroid gland parenchyma was unremarkable. Microscopic examination of the mass showed portions of an encapsulated tumour that was separated into nodules by broad, fibrous bands (Fig. 2). There was evidence of invasion into the tumour capsule, but not into the surrounding thyroid gland (Fig. 3). 


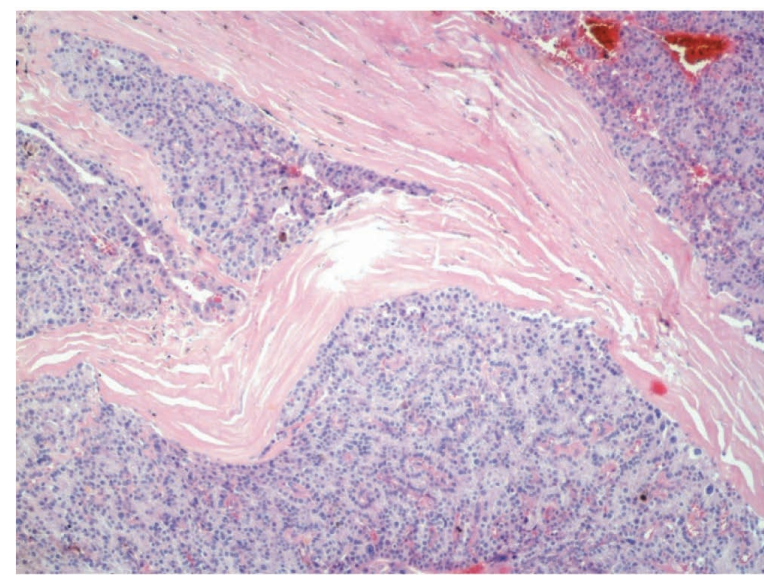

Fig. 2: Tumour intersected by broad fibrous bands. Haematoxylin and eosin $\times 100$.

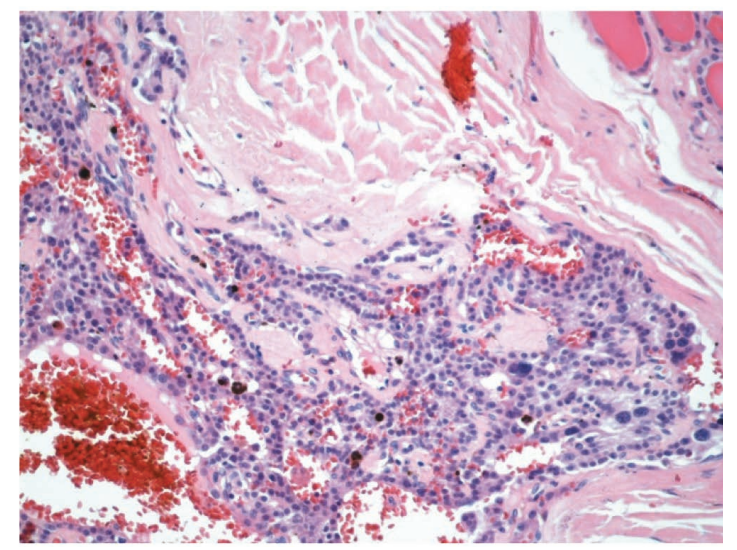

Fig. 3: Tumour invasion into capsule, without invasion into surrounding thyroid gland. Haematoxylin and eosin $\times 200$.

The neoplastic cells were disposed in trabeculae and solid nests within vascular stroma, and contained eosinophilic cytoplasm and round nuclei with conspicuous nucleoli. In some areas, there was marked nuclear pleomorphism, and the nuclei contained eosinophilic macronucleoli (Fig. 4).

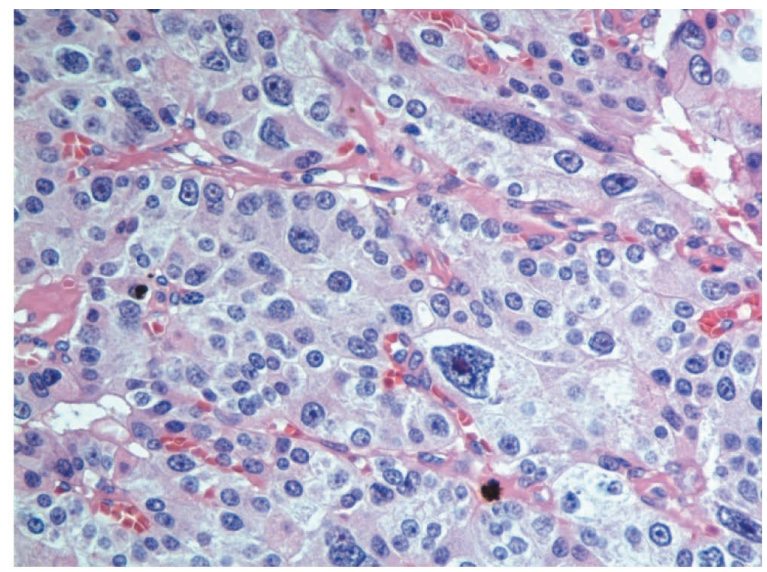

Fig. 4: Tumour cells containing pleomorphic nuclei with macronucleoli. Haematoxylin and eosin $\times 400$.
The mitotic rate was $<1$ mitotic figure per 10 high power fields. The overall histological picture was that of a parathyroid neoplasm, with the overall microscopic features exhibiting three of the four criteria required for a definitive diagnosis of carcinoma (3). However, given the identification of invasion of oesophageal muscle at surgery, a diagnosis of parathyroid carcinoma was rendered by the managing team. Sections of the remainder of the thyroid gland showed nodular hyperplasia. She was referred to the radiation oncologist for adjunctive therapy.

\section{DISCUSSION}

Parathyroid carcinoma accounts for $<1 \%$ of cases of primary hyperparathyroidism (4). The disease has been stated to be more common in Japan, where it accounts for $5 \%$ of the cases of primary hyperparathyroidism (2). It is less common in secondary or tertiary forms (5). It has been proposed that the occurrence in secondary hyperparathyroidism is the result of chronic stimulation of hyperplastic glands leading to malignant transformation of these glands (6). An estimated incidence of 5.73 per 10 million persons has been reported (6). The current case is the fourth case reported at our institution in over 40 years, with a total of four cases recorded in the archives of our pathology department from 1974 till present. Over the years, non-uniform criteria for histopathological differentiation between parathyroid adenoma and carcinoma may have resulted in our relatively low numbers.

The ratio of affected women to men has been reported in most series as 1:1 compared with other causes of primary hyperparathyroidism with a ratio of 3-4:1 (2). Another study reported that there was a slight predominance of cases in women (5). There have been a number of reports of cases of carcinoma arising from an adenoma or hyperplastic glands, and this has been proposed as an explanation for a slight predominance in women (2). Radiation exposure has usually occurred in these cases of transformation (7). However, others have found no evidence of malignant transformation of previous pathologic tissue (8), and this would explain why most series found equal gender distribution in PCA. The average age of presentation is in the fifth decade, 10 years younger than the typical patient with benign hyperparathyroidism (2). In a recent review of the Mayo Clinic experience, the average age was in the mid-fifties and the age range was 29 to 72 years (9). Our patient, a 67-year old woman, falls within this range.

Risk factors for PCA are unknown, but membership in a kin with either familial PCA or hyperparathyroidism-jaw tumour syndrome is a risk factor (6). Also, history of head and neck irradiation and chronic stimulation in secondary hyperparathyroidism are other risk factors (6).

The clinical course of PCA is unpredictable and indolent with recurrences occurring within 15 to 20 years after the first diagnosis (6). The most common symptoms of PCA are therefore those of hypercalcaemia: thirst, polydipsia, anorexia, severe weakness, constipation, renal colic, bone pain and vague 
abdominal pain (6). These are similar symptoms seen in benign hyperparathyroidism. Some of these symptoms are vague and might not be noticed by a patient and so might explain why our patient was reported as asymptomatic even though she was hypercalcaemic. In addition, while she did in fact have a calculus within the lower pole of the right kidney, it was non-obstructing and therefore explains why she did not present with renal colic. Thirty-four per cent of patients will present with a palpable neck mass which is uncommon in benign hyperparathyroidism. A palpable neck mass and a very high parathyroid hormone (PTH) level can be used to differentiate PCA from benign hyperparathyroidism (6). Unilateral vocal cord paralysis and hypercalcaemia are also very suspicious for PCA (6). In rare cases, a non-functioning PCA has been reported to present with a locally advanced mass (6). Our patient had a palpable neck mass. Parathyroid carcinoma usually grows slowly and metastasizes late (10). Lymph node metastatic disease is seen in $32 \%$ and lung metastatic disease is seen in $26 \%$ (10). Our patient had no evidence of metastatic disease.

Very high levels of PTH and severe hypercalcaemia are usually seen in PCA (6). The average calcium level seen in patients with PCA is $3.9 \mathrm{mmol} / \mathrm{L}$, compared to an average of 3 $\mathrm{mmol} / \mathrm{L}$ in adenomas (10). Ten per cent of cases of PCA have calcium levels less than $3.25 \mathrm{mmol} / \mathrm{L}$ and $75 \%$ of cases have a calcium level of $3.5 \mathrm{mmol} / \mathrm{L}$ or more (10). The index patient had a calcium level of $3.51 \mathrm{mmol} / \mathrm{L}$, consistent with levels seen most commonly in PCA. Recurrent hyperparathyroidism months after surgical resection is highly suggestive of recurrent disease, and recurrent hypercalcaemia has been reported as early as three months and as late as nine years after the initial surgery (10).

Ultrasound may be used to localize parathyroid tumours. Nonspecific features associated with PCA such as large size, inhomogenous appearance and irregular borders may alert the clinician to the possibility of PCA (6). Adenomas are smaller, homogenous and hypoechoic with smooth borders. Technetium-99 sestamibi scanning has been used successfully to localize PCA preoperatively but has been an unreliable tool to differentiate between an adenoma and PCA (6). It is also useful to detect metastatic PCA. In our case, both the ultrasound and sestamibi scan correctly diagnosed a parathyroid neoplasm but could not differentiate an adenoma from a carcinoma. Cytologically, it is impossible to differentiate between parathyroid tumours and tumours of the thyroid (10). In our case, the FNAC result was follicular variant of papillary carcinoma; therefore, FNAC of PCA is unnecessary (6).

The pathological diagnosis of PCA begins intraoperatively with the finding of an indurated mass invading surrounding structures such as the strap muscles, ipsilateral thyroid lobe, muscularis of the oesophagus, trachea and or recurrent laryngeal nerve (11). Unlike parathyroid adenomas, which are soft, circumscribed tumours with a thin fibrous capsule, parathyroid carcinomas are hard in consistency and often demonstrate a thick fibrous capsule. They may be either circumscribed or frankly invasive, and exhibit a grey-white colour, in contrast to the red-brown appearance of adenomas. The size of parathyroid carcinomas is variable, and they can range from as small as $1 \mathrm{~cm}$, to large tumours of several centimetres in diameter.

Microscopically, a thick capsule may be appreciated, if present. The cytological features of parathyroid carcinoma are similar to those of parathyroid adenoma (polygonal cells arranged in nests, solid sheets and trabecula) and so histopathological differentiation between the two may be difficult. However, there are criteria that have been suggested as guidelines for distinguishing between the two entities. Chang et al have suggested useful (absolute and relative) histopathological criteria for diagnosis of parathyroid carcinoma and have proposed that one absolute or four relative criteria be required to make a diagnosis of carcinoma rather than adenoma (3). The absolute criteria are: (i) invasion of surrounding soft tissue or surrounding vital structures, (ii) vascular invasion, (iii) perineural invasion and (iv) histologically documented regional or distant metastasis. The relative criteria are: (i) capsular invasion without extension into surrounding soft tissues, (ii) mitotic rate $>5$ mitotic figures per 10 high power fields, (iii) broad intratumoral fibrous bands separating the tumour into nodules, (iv) coagulative tumour necrosis, (v) diffuse sheet-like monotonous cells, (vi) diffuse cellular atypia and (vii) macronucleoli present in many tumour cells.

In the present case, the tumour showed only three of the required four relative criteria, but taken together with the gross evidence of invasion of oesophageal muscle at surgery, a diagnosis of parathyroid carcinoma was made.

In one study of 27 patients with PCA, 37\% of patients had fibrous bands, mitoses and vascular invasion; $26 \%$ had capsular invasion and $11 \%$ had trabeculae and lymphatic invasion (11). This study showed that the classic features are not always present in PCA. Also, mitotic figures and trabeculae architecture are seen in adenomas (11). Histologic criteria may not therefore definitively differentiate PCA from an adenoma and so frozen section may not be reliable unless the section reveals invasion of contiguous structures or lymph nodes. The morbidity and mortality associated with PCA are due to hypercalcaemia rather than tumour burden (11), so medical treatment of hypercalcaemia becomes important in patients with unresectable tumours. In patients in whom parathyroid carcinoma is suspected preoperatively and intraoperatively, there should be comprehensive soft tissue resection including an ipsilateral hemithyroidectomy (11). This may include excision of any overlying strap muscle, the paratracheal fibrolymphatic compartment and adjacent soft tissues, as required without sacrificing a normally functioning recurrent laryngeal nerve unless it is involved circumferentially by malignancy (11). Elective neck dissection is not indicated unless the nodes appear to be grossly involved by tumour (11). The local recurrence rate is lowered if adjuvant radiotherapy is given after initial surgery, independent of the type of surgery and disease stage (11). 
Parathyroid carcinoma has an indolent and progressive course. The average five-year survival of $85 \%$ is reported in various reports (11). Ten-year survival has been quoted as high as $77 \%(11)$.

\section{CONCLUSIONS}

There are no pathognomonic features to suggest parathyroid carcinoma. However, gross invasion at the time of surgery or recurrences along with the histopathological criteria can be used along with any other clinical clues to make a diagnosis of parathyroid carcinoma. The treatment should include a comprehensive soft tissue resection including hemithyroidectomy, and adjuvant radiation therapy.

\section{REFERENCES}

1. Taniegra ED. Hyperparathyroidism. Am Fam Physician 2004; 69: 3339.

2. Shane E. Parathyroid carcinoma. J Clin Endocrinol Metab [Internet] 2001 [cited 2014 Aug 24]; 86: 485-93. Available from: http://press. endocrine.org/doi/full/10.1210/jcem.86.2.7207

3. Chang Y-J, Mittal V, Remine S, Manyam H, Sabir M, Richardson T et al. Correlation between clinical and histological findings in parathyroid tumors suspicious for carcinoma. Am Surg 2006; 72: 419-26.

4. Marcocci C, Cetani F, Rubin MR, Silverberg SJ, Pinchera A, Bilezikian JP. Parathyroid carcinoma. J Bone Miner Res 2008; 23: 1869-80.
5. Sturniolo G, Gagliano E, Tonante A, Taranto F, Papalia E, Cascio R et al. Parathyroid carcinoma: case report. G Chir [Internet] 2013 [cited 2014 Aug 24]; 34: 170-2. Available from: http://www.ncbi.nlm.nih.gov/ pmc/articles/PMC3915582/

6. Adam MA, Untch BR, Olson JA Jr. Parathyroid carcinoma: current understanding and new insights into gene expression and intraoperative parathyroid hormone kinetics. Oncologist [Internet] 2010 [cited 2014 Oct 2]; 15: 61-72. Available from: http://www.ncbi.nlm.nih.gov/pmc/articles/ PMC3227890/

7. Busaidy NL, Jimenez C, Habra MA, Schultz PN, El-Naggar AK, Clayman GL et al. Parathyroid carcinoma: a 22-year experience. Head Neck 2004; 26: 716-26.

8. Schantz A, Castleman B. Parathyroid carcinoma. A study of 70 cases. Cancer [Internet] 1973 [cited 2014 Aug 24]; 31: 600-5. Available from: http://onlinelibrary.wiley.com/doi/10.1002/1097-0142 (197303)31:3<600::AID-CNCR2820310316>3.0.CO;2-0/full

9. Wynne A, Van Heerden J, Carney JA, Fitzpatrick LA. Parathyroid carcinoma: clinical and pathologic features in 43 patients. Medicine (Baltimore) [Internet] 1992 [cited 2014 Aug 24]; 71: 197-205. Available from: http://journals.lww.com/md-journal/Abstract/1992/07000/ Parathy roid_Carcinoma_Clinical_and_Pathologic.2.aspx

10. Holmes E, Morton D, Ketcham A. Parathyroid carcinoma: a collective review. Ann Surg [Internet] 1969 [cited 2014 Aug 24]; 169: 631-40 Available from: http://www.ncbi.nlm.nih.gov/pmc/articles/PMC1387475/

11. Clayman GL, Gonzalez HE, El-Naggar A, Vassilopoulou-Sellin R. Parathyroid carcinoma: evaluation and interdisciplinary management. Cancer [Internet] 2004 [cited 2014 Oct 12]; 100: 900-5. Available from: http://onlinelibrary.wiley.com/doi/10.1002/cncr.20089/full 